\title{
Lung Carcinoid Tumor
}

National Cancer Institute

\section{Source}

National Cancer Institute. Lung Carcinoid Tumor. NCI Thesaurus. Code C4038.

A neuroendocrine neoplasm that arises from the lung. It is characterized by the presence of uniform polyg onal cells with small or moderate amount of cytoplasm and inconspicuous nucleoli. The cells are usually arranged in organoid and trabecular patterns. It is classified as typical or atypical carcinoid tumor based on the number of mitotic figures and the absence or presence of necrosis. Atypical carcinoid tumors have a worse prognosis. 